\title{
Enhancing EEG Signals Recognition Using ROC Curve
}

\author{
Takashi Kuremoto $^{1}$, Yuki Baba ${ }^{2}$, Masanao Obayashi ${ }^{1}$, Shingo Mabu ${ }^{1}$, Kunikazu Kobayashi $^{3}$ \\ ${ }^{1}$ Graduate School of Science and Technology for Innovation, Yamaguchi University, Tokiwadai 2-16-1, \\ Ube, Yamaguchi 755-8611, JAPAN \\ ${ }^{2}$ Graduate School of Science and Engineering, Yamaguchi University, Tokiwadai 2-16-1, \\ Ube, Yamaguchi 755-8611, JAPAN \\ ${ }^{3}$ School of Information Science and Technology, Aichi Prefectural University, 1522-3 Ibaragabasama, \\ Nagakute, Aichi 480-1198, JAPAN \\ E-mail: \{wu,v033vk, m.obayas,mabu\}@yamaguchi-u.ac.jp, kobayashi@ist.aichi-pu.ac.jp
}

\begin{abstract}
Mental tasks, such as calculation, reasoning, motor imagery, etc., can be recognized by the pattern of electroencephalograph (EEG) signals. So EEG signal recognition plays an important role in brain-computer interaction (BCI). In this study, to enhance the ability of classifiers such as support vector machine (SVM), deep neural networks (DNN), k-nearest neighbor method ( $\mathrm{kNN}$ ), decision tree (DT), a feature extraction method is proposed using techniques of fast Fourier transform (FFT) and receiver operating characteristic (ROC) curve. In the proposed method, the raw EEG data was transformed into power spectrum of FFT at first, and then to find frequencies decided by area under curve (AUC) of ROC between the value of spectrums of different classes of metal tasks. Experiment results using benchmark data and BCI competition II data showed the effectiveness of the proposed method for all above classifiers.
\end{abstract}

Keywords: EEG, FFT, ROC, AUC, SVM

\section{Introduction}

The electronic potential signals of electrodes, which are arrayed on the surface of head, are measured by electroencephalograph and these signals are called as "Electroencephalogram", i.e., "EEG. EEG is also called brain wave data. When different metal tasks such as motor imagery, calculation, number counting, article considering, and so on, different patterns of EEG signals can be observed. So the analysis and recognition of EEG signal is one of the way to realized brain computer interface (BCI), or brain machine interface (BMI), i.e., people can control the machines or robots by their imaginations [1] [2]. Feature extraction of the EEG signal plays a very important role of mental task recognition to enhance the accuracy and there have been many studies of this theme [3] [4]. In [5], Obayashi et al. proposed to use the nonlinear normalized feature spaces of fast Fourier transformation (FFT) of EEG signals. And the method was improved by selecting the most characterized phases of raw EEG signals in our previous works [6].

In this paper, we propose a novel method using FFT and the value of area under curve (AUC) of receiver operating characteristic (ROC) curves to extract the feature of EEG signal and verified its effectiveness with kinds of classifiers: kernel-support vector machine (k-SVM), multi-layered perceptron (MLP), deep neural networks (DNN), k-nearest neighbor method $(\mathrm{kNN})$, and decision tree (DT). Benchmark data of EEG classification [7] and BCI competition II data [8] were used in the experiments to confirm the effectiveness of the proposed feature extraction method.

\section{Method}

To extract the feature vector space of EEG signal for mental task recognition, we propose to use FFT and ROC to find the limited frequencies which AUC values are high when two classes EEG data are compared in 
the training process, and the power spectrums of these frequencies are used as input vector of classifiers.

\subsection{Fast Fourier Transform (FFT)}

FFT is a kind of discrete Fourier transform (DFT), which is used for frequency analysis of signals in the field of signal processing, invented in 1965 by J. Cooley and J. Tukey at first, and improved continuously. The function of FFT is to show the power spectrum of different frequencies of the time series signals as same as the original Fourier transform but easily to be realized by computers. In this study, we used the FFT function provided by free software package $R$ [9] to process the raw EEG signals.

\subsection{Receiver operating characteristic (ROC) curve}

Assume that two classes data class A and class B have their probability density functions as shown in Fig. 1. The true positivity of class A will be the shadowed area $\alpha$ in the left and the false positives area $1-\beta$ in more shadowed area. As $\alpha$ and $1-\beta$ are plotted while sliding the threshold along the $\mathrm{x}$ axis, a graph as shown in Fig. 2 is obtained. The curve in this graph is called the ROC curve and it shows the divisibility of the two probability density functions. If two distributions completely overlap, the for any position of the threshold we have that $\alpha=1-\beta$.

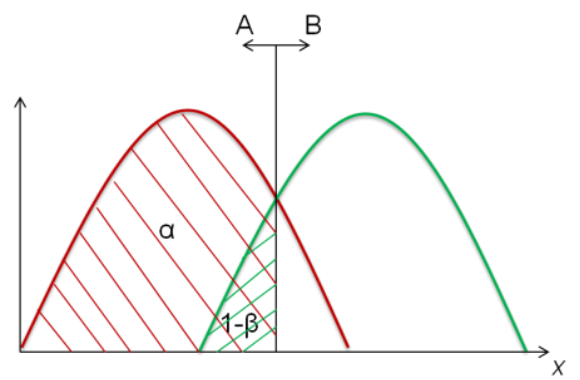

Fig. 1 Overlapping of the probabilities of two classes data.

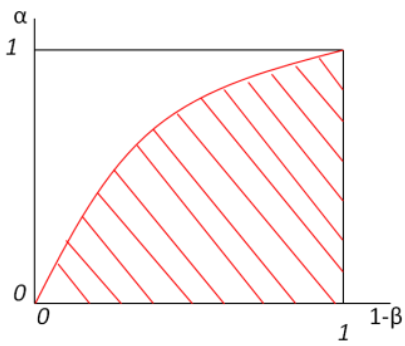

Fig. 2 AUC of a ROC curve
In Fig. 2, the area below the ROC curve is called "area under curve" (AUC). This value takes from 0.0 to 1.0 , and it is an indicator of the divisibility of the two distributions. If the value of AUC becomes to 0.5, two distributions completely overlapped. Conversely, when the value of AUC reaches 1.0 (or 0.0), it means that the two distributions are completely separated.

ROC was used to classify cDNA microarray successfully [10] and applied to EEG classification with wavelet transformation (WT) recently [11]. Here, we propose to use the value to AUC of ROC and FFT to distinguish the two classes EEG signals.

\section{Feature Extraction}

\subsection{Method of using FFT}

Suppose that an EEG signal $x_{m, n}(m=1,2, \ldots, M$, $n=1,2, \ldots, N)$ is given. $m$ indicates the number of channels, and $n$ indicates the number of samples in channel $m$. The procedure of the conventional method using FFT to extract the feature of EEG [6] is as follows.

Step 1 Divides the EEG signal of all the channels $m$ into multiple windows $l(l=1,2, \ldots, \mathrm{L})$ along the time domain

Step 2 Perform FFT on all of the divided windows, and let the result be $F_{m, l}$.

Step 3 Compare the power spectrum of the window $l$ of the channel $m$ to its adjacent window $l+1$, and calculate the difference $D_{m}(l)$ of the frequency spectrum.

$$
D_{m},(l)=\sum_{l=1}^{l=L-1}\left|F_{m, l+1}-F_{m, l}\right|
$$

Step 4 From Step 3, the maximum $D_{m}(l)$ in the channel $m$ is determined, and power spectrum in a region of 4 to $45 \mathrm{~Hz}$ in the frequency band of the window $l$ is used as a feature space for classifiers such as SVM, multi-layer perceptron (MLP), and so on.

\subsection{Method of using FFT and ROC Curve}

Let the input signals be $x_{k_{c}, m, n}(c=1$ or $2, k=1,2, \ldots$, $K)$, where $k$ indicates the $k$ th EEG signal of a set of EEG data, and $c$ indicates the class of mental task. $m$ and $n$ are the same as in section 3.1. The procedure of the novel method is as follows. 
Step 1 Perform FFT to all the EEG signals $x_{k_{c}, m, n}$ and let the result be $F_{k_{c}, m, p}$ where $p$ indicates the order number of frequencies.

Step 2 Obtain $P_{k_{1}, m, p}$ and $P_{k_{2}, m, p}$, which are two probability density functions of $F_{k_{c}, m, p}$ at $p$ frequency where class $c=1$ and 2 of $K$ signals of channel $m$.

Step 3 Calculate the ROC curve and its AUC $A_{m, p}$ of $P_{k_{1}, m, p}$ and $P_{k_{2}, m, p}$.

Step 4 Repeat Step 2 and Step 3 on all channels, a set $A_{m, p}$ of frequency $p$ in channel $m$ is obtained.

Step 5 Find $P$ points of frequencies which $A_{m, p}$ is high.

Step 6 Power spectrum $E_{m, p}(p=1,2, \ldots, P)$ of the unknown EEG signal are used to as input feature vector of a classifier.

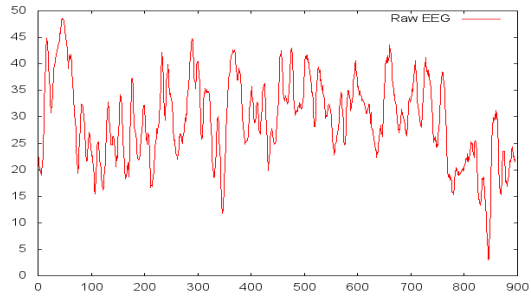

(a) The raw EEG data

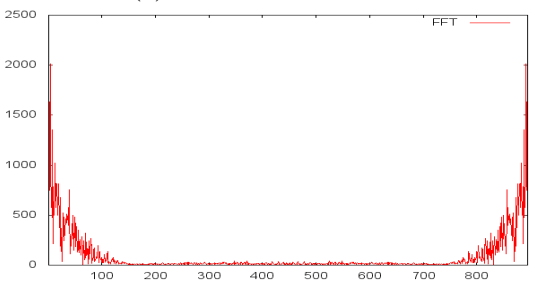

(b) FFT results

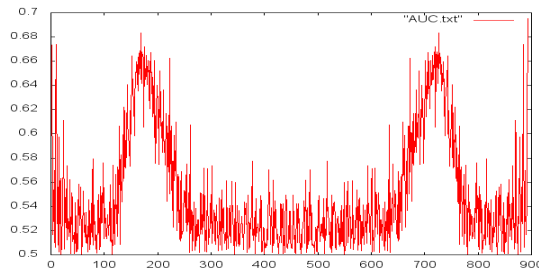

(c) AUC of FFT of 2 kinds of mental tasks

Fig. 3 The procedure to obtain AUC

The procedure of how AUC of FFT of 2 kinds of EEG data patterns is shown in Fig. 3 Frequencies with enough high values of AUC in Fig. 3 (c) are chosen as input features to classifiers.

\section{Recognition Experiment}

Mental task recognition experiments using EEG data and the methods introduced in Section 3 were performed. Two kinds of EEG data are used: (i) a benchmark data provided by Colorado State University [7] and (ii) BCI Competition II generated by Birbaumer [8]. In the case of (i), there are 5 kinds of mental tasks (see Table 1) were requested to subjects, and their EEG data were obtained 10 trials with 7 channels. Meanwhile in (ii), there are 2 data sets named "Ia" and "Ib" which are EEG data obtained by 2 kinds of tasks: Ia was asked to recognize visual objects displayed on the top or bottom of monitor and move a cursor up or down, and Ib with the same tasks but with not only visually presentation but also auditorily. Details of EEG data used in our experiments are listed in Table 2.

Table 1 Mental tasks in a benchmark database [7].

\begin{tabular}{l|l}
\hline Mental Task & Contents \\
\hline Baseline & Relaxing as much as possible \\
Multiplication & Calculating multiplication mentally. \\
Letter-composing & Considering the contents of the letter \\
Rotation & Imagining rotation of a 3-D object \\
Counting & Imagining writing a number in order \\
\hline
\end{tabular}

Table 2 EEG data used in the experiments.

\begin{tabular}{l|c|c|c|l|l}
\hline Database & $\begin{array}{l}\text { Mental } \\
\text { Tasks }\end{array}$ & $\begin{array}{l}\text { Data } \\
\text { Sets }\end{array}$ & $\begin{array}{l}\text { Chan } \\
\text { nels }\end{array}$ & $\begin{array}{l}\text { Samples } \\
\text { per } \\
\text { Channel }\end{array}$ & $\begin{array}{l}\text { Sampling } \\
\text { Freq. }\end{array}$ \\
\hline Benchmark & 5 & 10 & 7 & 2500 & 250 \\
[7] & 2 & $135 / 133$ & 6 & 896 & 256 \\
Ia [8] & 2 & 100 & 7 & 1152 & 256 \\
Ib [8] & \multicolumn{1}{l}{} \\
\hline
\end{tabular}

Table 3 Recognition rate (\%) of Benchmark data [7]

\begin{tabular}{c|c|c}
\hline Methods & $\begin{array}{l}\text { FFT with ROC } \\
\text { (proposed) 140 dim. }\end{array}$ & $\begin{array}{l}\text { The change of } \\
\text { FFT 140 dim. }\end{array}$ \\
\hline kSVM & $\mathbf{9 7 . 5}$ & 59.6 \\
\hline MLP & $\mathbf{5 5 . 0}$ & 49.6 \\
\hline kNN & $\mathbf{7 3 . 3}$ & 55.9 \\
\hline DNN & $\mathbf{9 5 . 4}$ & 61.7 \\
\hline DT & $\mathbf{5 0 . 0}$ & 34.5 \\
\hline
\end{tabular}

Table 4 Recognition rate (\%) of Ia data [8]

\begin{tabular}{c|c|c}
\hline Methods & $\begin{array}{c}\text { FFT with ROC } \\
\text { (proposed) 1120 dim. }\end{array}$ & $\begin{array}{c}\text { The change of } \\
\text { FFT 1120 dim. }\end{array}$ \\
\hline kSVM & $\mathbf{9 1 . 2}$ & 59.0 \\
\hline MLP & $\mathbf{7 0 . 9 .}$ & 50.0 \\
\hline kNN & $\mathbf{5 5 . 8}$ & 55.5 \\
\hline
\end{tabular}




\begin{tabular}{c|c|c}
\hline DNN & $\mathbf{8 6 . 1}$ & 62.1 \\
\hline DT & $\mathbf{7 3 . 2}$ & 43.2 \\
\hline
\end{tabular}

Kernel SVM (k-SVM), MLP, kNN, DNN, DT were used as classifiers. Functions Kernlab, nnet, class, $\mathrm{h} 2 \mathrm{o}(+\mathrm{JavaVM})$, rpart corresponding to the above classifiers, and ROC package in R [9] were utilized in our experiments.

The recognition results by the different classifiers and feature extraction methods for EEG signals are shown in Tables 3, 4, and 5. The classification rates of the conventional method [6] (FFT) were lower than the recognition rate of the proposed method (FFT+ROC) in all cases of EEG data. Specially, kSVM showed the best performance among these classifiers in the case of the proposed feature extraction method.

Table 5 Recognition rate (\%) Ib data [8]

\begin{tabular}{c|c|c}
\hline Methods & $\begin{array}{c}\text { FFT with ROC } \\
\text { (proposed) 1120 dim. }\end{array}$ & $\begin{array}{c}\text { The change of } \\
\text { FFT 110 dim. }\end{array}$ \\
\hline kSVM & $\mathbf{7 7 . 7}$ & 53.9 \\
\hline MLP & 49.1 & $\mathbf{5 3 . 4}$ \\
\hline kNN & $\mathbf{5 7 . 8}$ & 48.3 \\
\hline DNN & $\mathbf{7 5 . 3}$ & 49.3 \\
\hline DT & $\mathbf{5 5 . 6}$ & 48.0 \\
\hline
\end{tabular}

Table 6 Recognition rate (\%) of BCI Competition II [8]

\begin{tabular}{|c|c|c|}
\hline Data & $\begin{array}{l}\text { Kernel SVM using } \\
\text { proposed feature } \\
\text { extraction method }\end{array}$ & $\begin{array}{l}\text { Wavelet transform } \\
\text { and fuzzy logic } \\
\text { system [11]. }\end{array}$ \\
\hline Ia & 91.23 & 90.10 \\
\hline $\mathrm{Ib}$ & 77.65 & 56.67 \\
\hline
\end{tabular}

The highest recognition rates of kSVM using our feature extraction method for Ia and $\mathrm{Ib}$ are $91.23 \%$ and $77.65 \%$, higher than the best classification rates $90.10 \%$ and $56.67 \%$ of T. Nguyen et al. [11] respectively (Table $6)$.

\section{Conclusion}

In this paper, we proposed a feature extraction method for EEG pattern recognition. The proposed method uses the AUC value of ROC curves of FFT results for two classes EEG signals to decide the feature vector space for classifiers. Classification experiments were performed using the Gaussian kernel SVM (kSVM) to confirm the proposed method. As the result of the experiments, the proposed method showed its higher recognition ability than the conventional methods including the conventional FFT feature extraction method and others. We also used ROC of raw EEG data and ROC of wavelet transform (WT) as the input of kSVM, MLP, kNN, DNN, and DT, and experiment results showed that the proposed method, i.e., features of EEG data extracted by ROC of FFT, were superior to the conventional feature extraction method.

\section{Acknowledgements}

This work was supported by JSPS KAKENHI Grant No. 26330254 and No.25330287.

\section{References}

1. N. Birbaumer, L. G. Cohen, Brain-computer interfaces: communication and restoration of movement in paralysis, Journal of Physiology, Vol. 579, pp. 621-636, 2006

2. F. Lotte, M. Congedo, A.Lecuyer, F. Lamarche, B. Arnaldi, A review of classification algorithms for EEGbased brain-computer interfaces, Journal of Neural Engineering, Vol. 4, pp. 24-48, 2007

3. N. Jrad, M. Conedo, Identification of spatial and temporal features of EEG, Neurocomputing, Vol. 90, pp.66-71, 2012

4. A.S. Al-Fahoum, A. A. Al-Fraihat, Methods of EEG signal features extraction using linear analysis in frequency and time frequency domains, ISRN Neuroscience, Vol. 2014, 7 pages, 2014

5. M. Obayashi, K. Watanabe, T. Kuremoto, K. Kobayashi, Development of a brain computer interface using inexpensive commercial EEG sensor with one-channel, Proceedings of the $17^{\text {th }}$ International Symposium on Artificial Life and Robotics (ISAROB 2012), pp. 714-717, 2012

6. T. Kuremoto, Y. Baba, M. Obayashi, S. Mabu, K. Kobayashi, To extraction the feature of EEG signals for mental task recognition, Proceedings of $54^{\text {th }}$ Annual Conference of the SICE, pp. 353-358, 2015

7. Colorado State University, Brain-Computer Interfaces Laboratory: http://www.cs.colostate.edu/eeg/

8. BCI competition II: http://www.bbci.de/competition/ii/\#datasets

9. The R Project for Statistical Computing: https://www.rproject.org/

10. H. Mamitsuka, Selecting features in microarray classification using ROC curves, Pattern Recognition, Vol. 39, Issue 12, pp. 2393-2404, 2006

11. T. Nguyen, A. Khosravi, D. Creighton, S. Nahavandi, EEG signal classification for BCI applications by wavelets and interval type-2 fuzzy logic systems, Expert Systems with Applications, Vol. 42, pp. 4370-4380, 2015 